\title{
Connecting structure and function from organisms to molecules in small-animal symbioses through chemo-histo-tomography
}

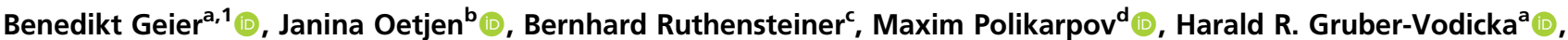 \\ and Manuel Liebeke ${ }^{a, 1}$ (iD

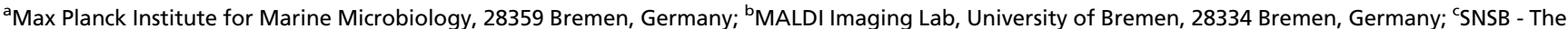
Bavarian State Collection of Zoology, 81247 Munich, Germany; and ${ }^{\mathrm{d} E u r o p e a n}$ Molecular Biology Laboratory, Hamburg Unit c/o Deutsches
\end{abstract} \\ Elektronen-Synchrotron, 22607 Hamburg, Germany
}

Edited by Margaret McFall-Ngai, University of Hawaii at Manoa, Honolulu, HI, and approved May 24, 2021 (received for review January 7, 2021)

\begin{abstract}
Our understanding of metabolic interactions between small symbiotic animals and bacteria or parasitic eukaryotes that reside within their bodies is extremely limited. This gap in knowledge originates from a methodological challenge, namely to connect histological changes in host tissues induced by beneficial and parasitic (micro)organisms to the underlying metabolites. We addressed this challenge and developed chemo-histo-tomography (CHEMHIST), a culture-independent approach to connect anatomic structure and metabolic function in millimeter-sized symbiotic animals. CHEMHIST combines chemical imaging of metabolites based on mass spectrometry imaging (MSI) and microanatomy-based micro-computed X-ray tomography (micro-CT) on the same animal. Both high-resolution MSI and micro-CT allowed us to correlate the distribution of metabolites to the same animal's three-dimensional (3D) histology down to submicrometer resolutions. Our protocol is compatible with tissue-specific DNA sequencing and fluorescence in situ hybridization for the taxonomic identification and localization of the associated micro(organisms). Building CHEMHIST upon in situ imaging, we sampled an earthworm from its natural habitat and created an interactive 3D model of its physical and chemical interactions with bacteria and parasitic nematodes in its tissues. Combining MSI and micro-CT, we present a methodological groundwork for connecting metabolic and anatomic phenotypes of small symbiotic animals that often represent keystone species for ecosystem functioning.
\end{abstract}

X-ray micro-CT imaging | 3D reconstruction | metabolomics | symbiosis | multimodal mass spectrometry imaging

$E^{a}$ arthworms represent a prime example of a keystone species (1) that experiences constant chemical interactions with bacteria (2), fungi (3), plants, and small invertebrates (4) across soil ecosystems. Even within their tissues earthworms harbor symbiotic microbes (5) and small animal parasites (6) that trigger internal metabolic responses such as innate immunity.

Unlike the metabolites used by earthworms for the digestion of leaf litter (7), the metabolites involved in the chemical interactions between earthworms and their associated (micro)organisms are unknown. For instance, most lumbricid earthworms harbor speciesspecific bacteria in their excretory organs. Still, it is unclear whether the symbionts complement the host through vitamins or detoxification of nitrogenous waste products (8). In addition to mutualistic bacteria, nematodes infest the muscles, blood vessels, and excretory organs in over 10 earthworm species (9). Investigating the metabolic interactions between earthworms and their associated partners could allow us to unravel how earthworms have become the engineers and janitors of soil ecosystems across the globe $(4,10)$.

The sum of mutualistic, commensal, and pathogenic interactions results in a unique anatomic and, in particular, metabolic phenotype for nearly every host individual $(11,12)$. Resolving this variability, in situ imaging of both metabolic and cellular phenotypes of the same host organ revealed metabolites which drive metabolic heterogeneity of the symbiotic partners $(13,14)$.
For instance, correlative metabolite imaging of the respiratory epithelia in a symbiotic invertebrate showed that within tens of micrometers the same species of intracellular bacterial symbionts produces different membrane lipids (13). Notably, metabolic interactions between animals and their microbes are not restricted to symbiotic tissues (15). Along the gut-brain axis, microbial metabolites produced in the gut can affect tissues across the host reaching the brain (16). Therefore, extending correlative chemical imaging into three-dimensional (3D) approaches can be crucial for capturing the distribution of metabolites involved in symbiotic interactions occurring in animal hosts (17).

Previous studies have addressed this methodological challenge. They combined nondestructive magnet resonance tomography with metabolite imaging, so-called matrix-assisted laser desorption/ionization (MALDI) mass spectrometry imaging (MSI), which enabled the colocalization between the distribution of individual metabolites and the 3D anatomy of organs (18) and pathogenic abscesses (19-21). The spatial resolution used in these approaches was ideal for imaging animals with millimeter-sized organs such as mice. However, the majority of

\section{Significance \\ Metabolites mediate the establishment and persistence of most interkingdom symbioses. Still, to pinpoint the metabolites each partner displays upon interaction remains the biggest challenge in studying multiorganismal assemblages. Addressing this challenge, we developed a correlative imaging workflow to connect the in situ production of metabolites with the organ-scale and cellular three-dimensional distributions of mutualistic and pathogenic (micro)organisms in the same host animal. Combining mass spec- trometry imaging and micro-computed X-ray tomography pro- vided a culture-independent approach, which is essential to include the full spectrum of naturally occurring interactions. To introduce the potential of combining high-resolution tomography with me- tabolite imaging, we resolved the metabolic interactions between an invertebrate host, its symbiotic bacteria, and tissue parasites at unprecedented detail for model and nonmodel symbioses.}

Author contributions: B.G. and M.L. designed research; B.G. and M.P. performed research; J.O. and B.R. contributed new reagents/analytic tools; B.G., H.R.G.-V., and M.L. analyzed data; and B.G. and M.L. wrote the paper.

The authors declare no competing interest.

This article is a PNAS Direct Submission.

This open access article is distributed under Creative Commons Attribution-NonCommercialNoDerivatives License 4.0 (CC BY-NC-ND).

See online for related content such as Commentaries.

${ }^{1}$ To whom correspondence may be addressed. Email: bgeier@mpi-bremen.de or mliebeke@mpi-bremen.de.

This article contains supporting information online at https://www.pnas.org/lookup/suppl/ doi:10.1073/pnas.2023773118/-/DCSupplemental.

Published June 28, 2021. 
animals used as symbiosis models (22), apart from medical studies, have body sizes of only a few millimeters to centimeters. Imaging their 3D anatomy and associated (micro)organisms together with their metabolite profiles requires each imaging technique to achieve micro- to nanometer resolutions. To assess the metabolic interactions taking place at the interface between host tissue and associated (micro)organisms in situ imaging of the symbiotic tissues is essential.

The integration of micro-computed tomography (micro-CT) and MALDI-MSI technologies is an emerging approach to image an animal's 3D histology and spatial chemistry at micrometer scales (23). Micro-CT is a noninvasive approach allowing X-ray imaging of 3D histology, and unlike magnetic resonance tomography microCT can reach subcellular resolution (24-27). For metabolite imaging, MALDI-MSI techniques have also reached subcellular resolutions $(28,29)$. For imaging animal models, both techniques usually have been applied separately, following two different imaging workflows. The principal obstacle is that MSI requires tissue sectioning and thus cannot be applied before nondestructive 3D imaging. Conventional micro-CT, on the other hand requires chemical contrasting of soft tissues (30), which would change the chemistry of the sample and interfere with subsequent MSI (31).

Here we present chemo-histo-tomography (CHEMHIST) that combines MALDI-MSI and micro-CT, providing a framework for imaging both the spatial chemistry and 3D microanatomy of the same small symbiotic animal. Our objective was to make CHEMHIST also applicable to animals directly sampled from their natural habitat through state-of-the-art in situ imaging and tissue-specific metagenomic DNA sequencing within the same pipeline. CHEMHIST provides an up to two orders of magnitude higher resolution than previous correlative 3D MALDIMSI approaches. This advance allowed us to take an earthworm from the environment and create a 3D atlas of its chemical and physical interactions with bacteria and nematodes naturally occurring inside its tissues.

\section{Results and Discussion}

We used earthworms as target organisms for developing our correlative high-resolution tomography and metabolite imaging workflow. They are easily accessible and their diverse associations with parasites and microbes result in phenotypic heterogeneity that demands correlative imaging at scales from millimeters to micrometers.

CHEMHIST Workflow for Creating a Multimodal 3D Atlas. CHEMHIST consists of three major steps. First, we physically divided the sample that was snap-frozen in its habitat for the different fixation and sample preparation procedures of MSI and micro-CT (Fig. 1A). Second, we imaged the samples with MSI and micro-CT, resolving organ-sized structures measuring tens to hundreds of micrometers, instead of using time-consuming high-spatial-resolution measurements. This trade-off between spatial resolution and imaging speed allowed us to record the 3D anatomy from tissue blocks and the distribution of metabolites across tissue sections of the wholeanimal width and reconstruct our CHEMHIST 3D model (Fig. 1B). In the third step, we complemented our organ-scale CHEMHIST overview with high-spatial-resolution measurements by remeasuring specific regions of interest. From the combined 3D overview, we determined tissue regions that were colonized by microbes or parasites and exhibited a specific chemistry, to guide high-resolution MSI and micro-CT to these regions. By combining multiscale imaging for micro-CT (25) and MSI (32) we could screen for micrometer-scale physical and chemical interactions inside a millimeter-sized animal without producing excessive amounts of data. Because we used the CHEMHIST 3D model as an overview to guide the high-resolution measurements, we referred to the $3 \mathrm{D}$ model as an atlas (33).
To apply MSI and micro-CT to the same animal, here to an earthworm (Lumbricus rubellus) (34), we had to treat the sample in a manner that preserves both morphology and spatial distribution of metabolites but without one technique interfering with the other. For CHEMHIST, snap freezing provided a trade-off between preserving enough anatomic details for micro-CT without modifying the distribution of metabolites for MSI. For micro-CT and MSI we divided our frozen sample into two sample types: tissue blocks and tissue sections. The tissue blocks of 1- to 3-mm thickness were trimmed off the frozen sample with a razor blade and tissue sections of $16-\mu \mathrm{m}$ thickness were sectioned off each tissue block with a cryotome (Fig. $1 A$ ). We obtained tissue blocks for micro-CT and tissue sections for MSI. Additionally, we stored consecutive tissue sections from in between the tissue blocks for high-resolution MSI and spatially targeted metagenomics sequencing. Obtaining both sample types in an alternating manner provided sample pairs of one tissue block for micro-CT and one adjacent tissue section for MSI (Fig. $1 A$ ). We chose cross-sections over longitudinal sections, which was critical to apply our alternating sectioning approach and obtain cubic tissue samples to fully take advantage of 3D imaging with micro-CT. Additionally, the smaller cutting plane of cross- instead of longitudinal sections resulted in higher-quality tissue sections as the tissue was not stabilized with chemical fixatives. Sharing the same sectioning interface, each tissue section anatomically and chemically matched its adjacent tissue block, which allowed us to precisely correlate micro-CT and MSI data despite segregating the tissues.

To generate the anatomic $3 \mathrm{D}$ atlas, we imaged each of the five tissue blocks with micro-CT at a 4.4- $\mu \mathrm{m}$-volume pixel (voxel) size. From the five micro-CT datasets, we virtually reconstructed a $3 \mathrm{D}$ anatomy model of the specimen (Fig. $1 B$ and SI Appendix, Fig. S2). To gain an overview on how metabolites are distributed throughout the sample, we imaged four cryo-sections with MALDI time-of-flight (TOF)-MSI at a $25-\mu \mathrm{m}$ pixel size (SI Appendix, Fig. S3). After MSI we imaged the histology with bright-field microscopy and we specifically labeled bacterial cells with fluorescence in situ hybridization (FISH) in each of the four tissue sections (13) (Fig. $1 B$ and SI Appendix, Fig. S4). Notably, MSI approaches using MALDI are not yet capable of resolving single bacterial cells and we therefore relied on the correlative FISH signals to establish a correlation to the metabolite signals. To complete the 3D CHEMHIST atlas, we coregistered the two-dimensional (2D) imaging MSI, FISH, and bright-field microscopy datasets into the anatomic 3D model (Fig. $1 B$ and SI Appendix, Fig. S2).

\section{CHEMHIST Provides a Cross-Kingdom Link between Spatial Chemistry} and 3D Anatomy. Organ-specific anatomy as well as chemistry are inherently linked to organ functioning, such as in movement, digestion, or signal transduction. Each metabolite imaged with MSI is identified by a mass-to-charge ratio $(\mathrm{m} / \mathrm{z})$. To study the association between organ-specific metabolites and 3D anatomy of the earthworm, we grouped all $\mathrm{m} / \mathrm{z}$ images by spatial distribution and matched each image group to the respective organ we reconstructed from the micro-CT data. We used unsupervised spatial clustering to group similarly distributed metabolites (35) and we used manual segmentation and thresholding of gray values to reconstruct individual organs from the 3D micro-CT data (Fig. $1 C$ and $D$ ). Both analyses allowed us to delineate overall chemical and anatomical features and gain an overview on the anatomic 3D structure and metabolic function of each organ.

With MSI we located metabolites in individual organs in situ at a hundred- to thousand-fold increased precision as opposed to manual dissection of each organ (Fig. $1 F$ ). For example, among the metabolites located in the musculature (Fig. $1 F$ ), we found lombricine, an annelid-specific energy storage metabolite that was highly abundant in the musculature of the animal (36) (SI Appendix, Table S1). Another metabolite with organ-specific localization is protoporphyrin, a pigment only located in the dorsal part 
A

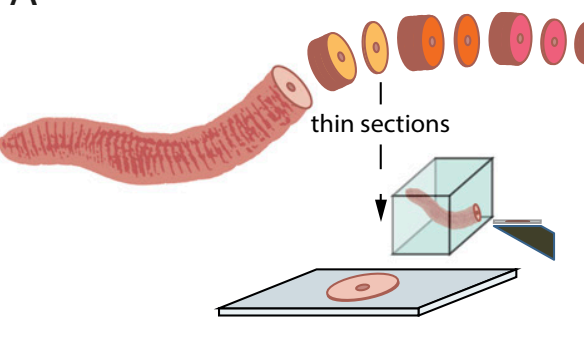

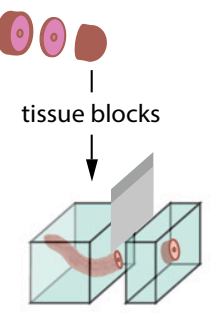

D

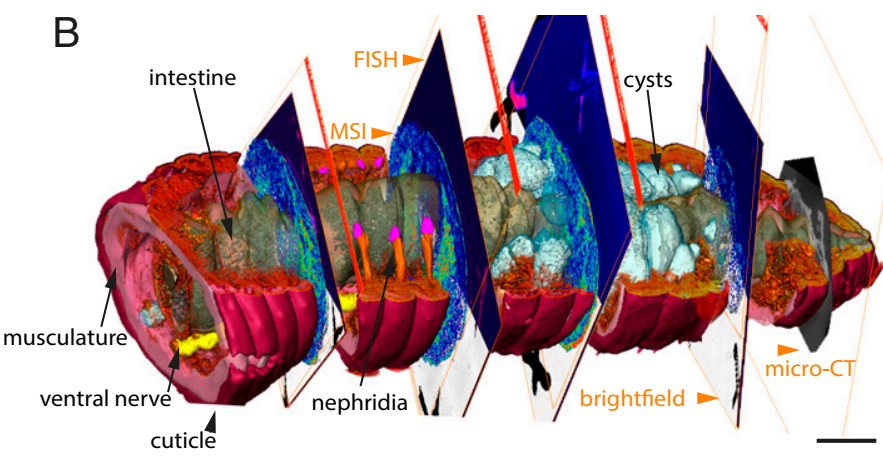

C

segmented surface model (micro-CT data) segmentation maps (MALDI-TOF-MSI)
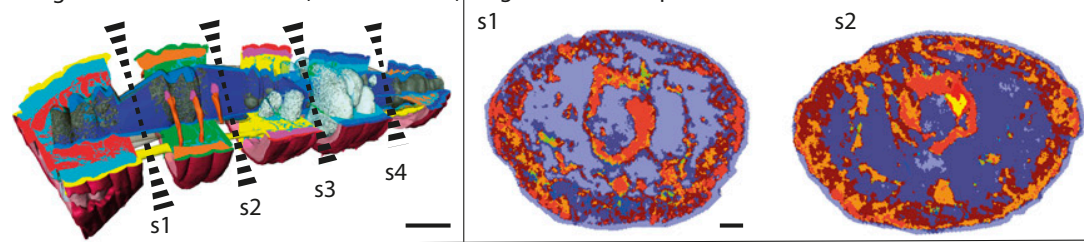

s3

s4

E surface models

musculature

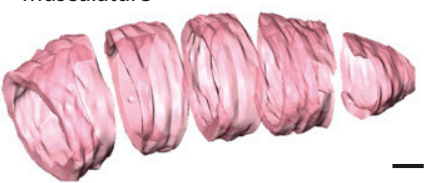

gut content

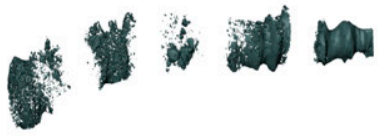

nematode cysts
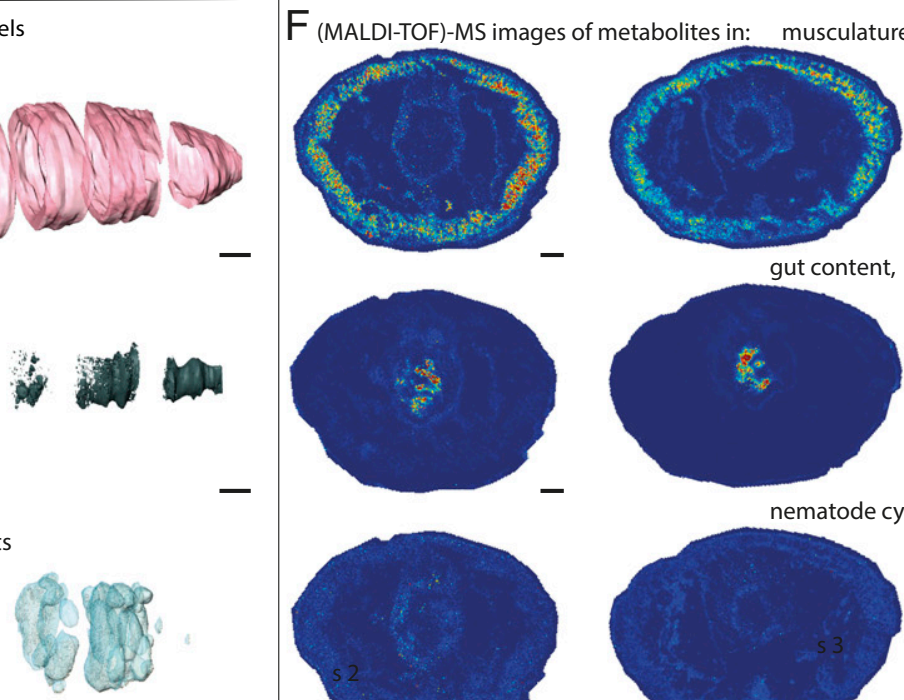

gut content, $\mathrm{m} / \mathrm{z} 177.919 \pm 0.200 \mathrm{Da}$
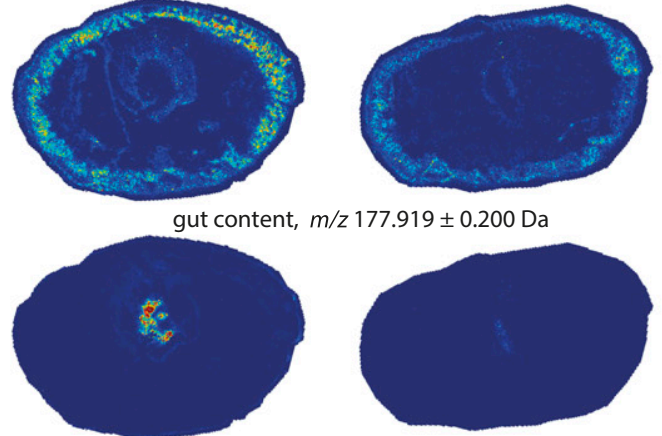

nematode cysts, $\mathrm{m} / \mathrm{z} 262.177 \pm 0.200 \mathrm{Da}$
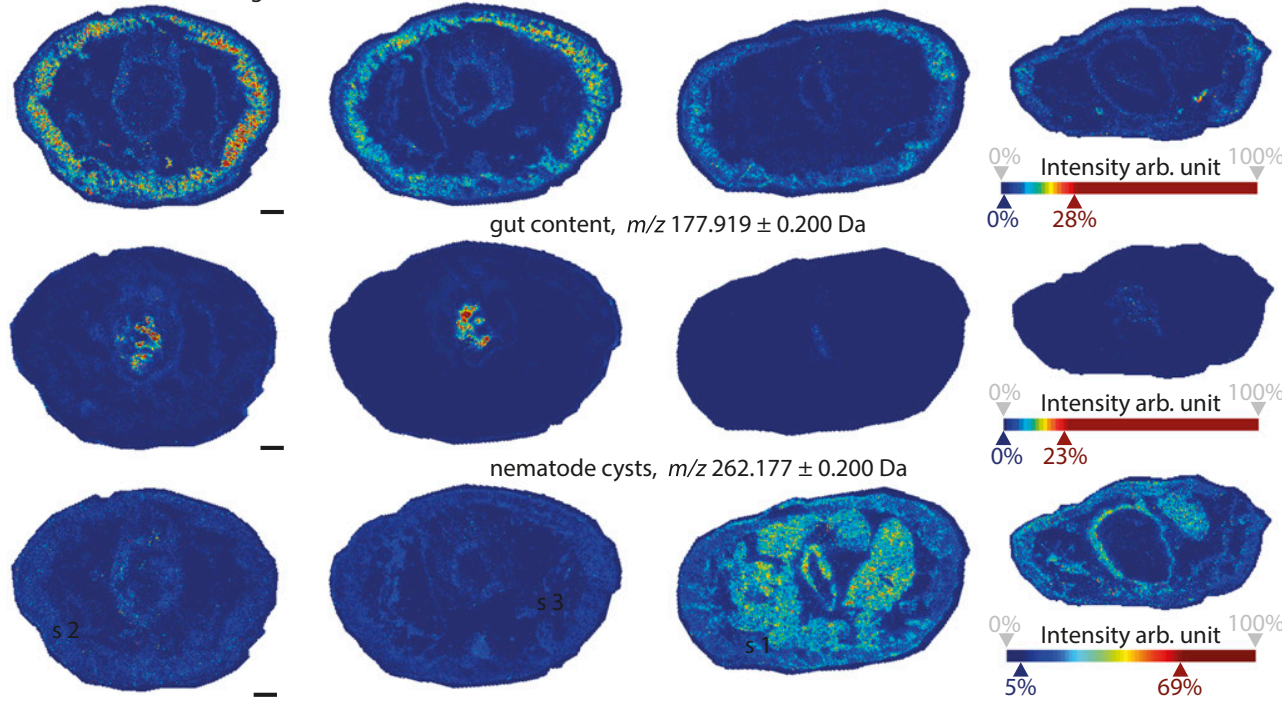

Fig. 1. CHEMHIST revealed organ-specific chemistry in the posterior segments of an earthworm. $(A)$ Division of the animal into alternating tissue sections for metabolite imaging and microscopy and tissue blocks for tomography. (B) Three-dimensional CHEMHIST atlas at organ scale (imaging techniques: orange; organ labels: black). (C) Segmentation of the 3D micro-CT data (surface models, semiautomatic segmentation) and (D) 2D MALDI-TOF-MSI data to delineate the spatial chemistry across all four sections applying unsupervised spatial metabolite clustering. Each metabolite cluster is highlighted with a separate color (i.e., nematode cyst cluster in green). Sectioning planes of sections $s 1$ to s4 indicated in $C$ by dashed wedge. ( $E$ ) Examples of surface models of individual organs and $(F)$ individual metabolites located in the organ shown in $E$ throughout sections s1 to s4. Color bars in $F$ show relative ion abundances for MS images, normalized to the total ion counts. (Scale bars in $B, C$, and $E$ : 2,000 $\mu \mathrm{m}$ and in $F$ : $500 \mu \mathrm{m}$.) Two-dimensional scale bars in $3 D$ models as approximate scale.

of the epidermis in lumbricid earthworms (37) (SI Appendix, Table S1).

The critical advantage of our sequential 2D MSI and 3D micro-CT approach is the potential to detect local deviations in the metabolite composition in correlation to an organ's 3D structure. For instance, the earthworm's intestine appeared as a homogeneously filled tube but the metabolites varied considerably along the length axis of the specimen (Fig. $1 E$ and $F$ ).

Combining micro-CT with MSI also allowed us to identify discontinuous organs and anatomic abnormalities throughout the animal that indicated metabolic heterogeneity. For instance, the nephridia, which harbor symbiotic bacteria $(5,8)$, occur pairwise in each segment and were not present in each tissue section used for MSI (Fig. 2). The 3D CHEMHIST atlas helped to locate histological structures that originated from sectioning planes through the nephridia (Figs. $1 B$ and $2 A$ ). Applying correlative FISH microscopy on the same tissue sections after MSI (13) with probes targeting eubacteria enabled us to locate bacterial accumulations across each tissue section and identify the symbiotic tissues of the nephridia (Fig. $2 A, D$, and $E$ ). This approach enabled us to use the fluorescence signals of the labeled bacteria to screen for metabolites that spatially correlated to the bacterial accumulations in the nephridia identified from the 3D atlas (Fig. $2 B$ and $C$ and $S I$ Appendix, Fig. S4) (13).

Guiding High-Resolution MSI and Micro-CT to Visualize Host-Parasite Interactions with CHEMHIST. The 3D CHEMHIST atlas of a fieldcollected earthworm also facilitated the analysis of structures in detail that are not part of an earthworm's anatomy blueprint. Our analysis of the 3D atlas indicated 20- to 30- $\mu \mathrm{m}$ - (in diameter) sized parasitic worms encysted in the earthworm tissues. To showcase our combination of micrometer-scale metabolite 

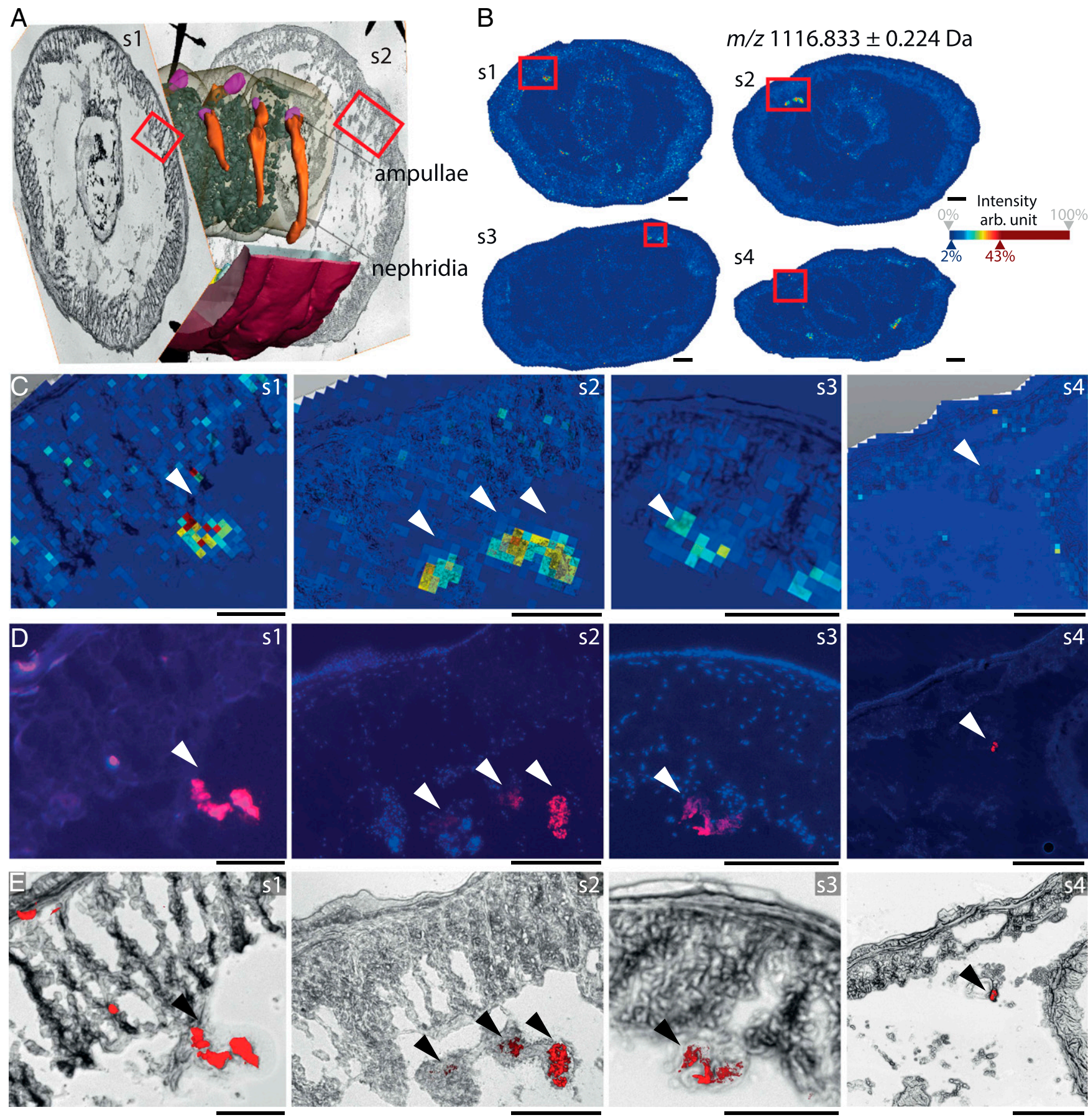

Fig. 2. Bacterial cells within organs have unique molecular fingerprints. $(A)$ The $3 D$ model shows the surface reconstruction of the nephridia, including the bacteria-containing ampullae of the second tissue block between tissue sections $s 1$ and $s 2$. (B) The ion images of whole tissue sections show an unidentified metabolite $\mathrm{m} / \mathrm{z} 1,116.833$, with the highest colocalization between MALDI-MSI and FISH signals. Red boxes in s1 to s4 indicate magnified areas, shown as MSI and bright-field overlay in C. (D) FISH microscopy images and (E) FISH and bright-field overlay with bacteria labeled in red. Color bar in $B$ shows relative ion abundances for MS images shown in $B$ and $C$. (Scale bars of whole tissue sections s1 to $s 4$ in $B: 500 \mu \mathrm{m}$ and in magnifications in $C-E$ : $250 \mu \mathrm{m}$.)

imaging, nanometer-scale histology, and metagenomics sequencing, we visualized the metabolic and anatomic phenotypes of these small worms and surrounding host tissues in situ (Fig. 3).

The cysts containing the nematodes occurred irregularly, increasing in size and density toward the posterior of the earthworm (Figs. $1 B$ and $3 A$ and SI Appendix, Fig. S6). By specifically targeting cyst tissues for DNA sequencing and phylogenetic analysis, we identified the nematodes as Rhabditis maupasi (SI Appendix, Fig. $\mathrm{S} 5)$. The species resides as parasites in the nephridia and coelomic cavities in common earthworm species such as Lumbricus terrestris, L. rubellus, Allobophora longa, and Allobophora turgid (38).

In earthworms, these cysts are called brown bodies and are produced to encapsulate and degrade organic debris, microbes, or parasites through reactive oxygen species $(39,40)$. To visualize the nanometer-scale $3 \mathrm{D}$ histology during the degradation process of single nematodes in the brown bodies, we used synchrotron radiation-based (SR) micro-CT. This high-resolution micro-CT technique allowed us to rescan cyst areas with high 

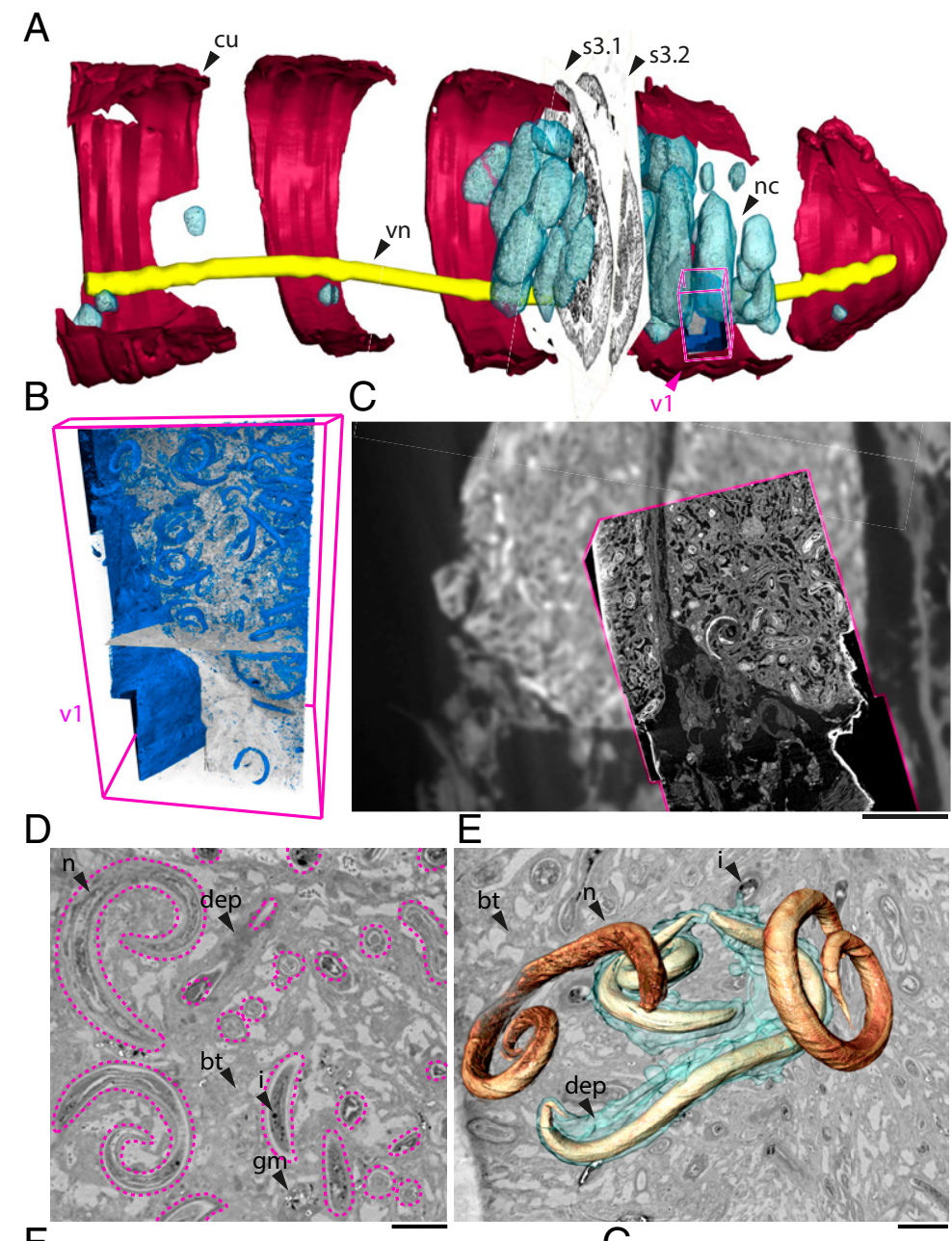

E

$\mathrm{F}$
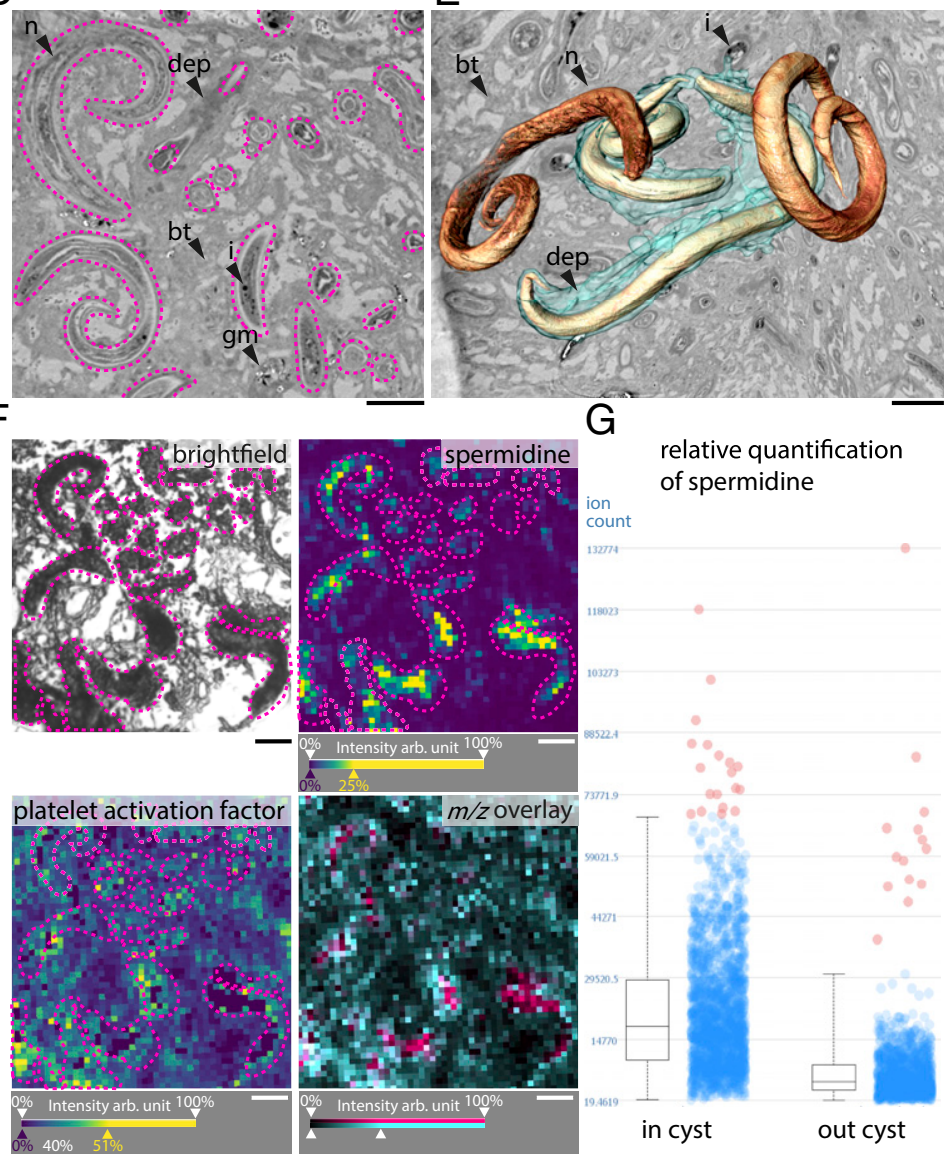

G

relative quantification of spermidine

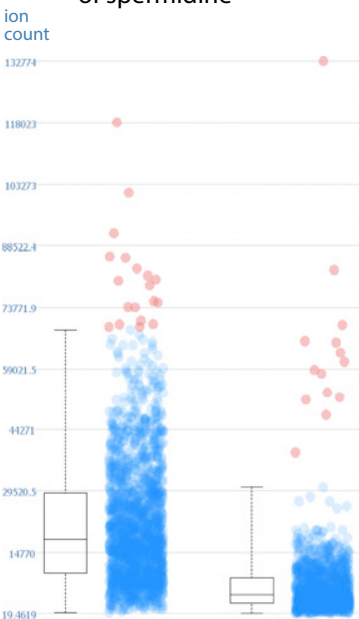

in cyst

out cyst

Fig. 3. Using the 3D CHEMHIST atlas to guide high-resolution imaging of the interactions between earthworm and parasitic nematodes. (A) Micro-CT model with the coregistered MSI sections (s3.1 and s3.2) and the tissue volume (v1) imaged with high-resolution SRmicro-CT. (B) Isosurface 3D rendering of the nematodes (blue) and a virtual sectioning plane (xy) through the SRmicro-CT image stack (v1). (C) Overlay of the micro-CT and SRmicro-CT (magenta outline, xy plane) to show the increased resolution and detail gained with SRmicro-CT. (D) Virtual plane through the SRmicro-CT data shows sections of nematodes in the cysts (magenta outlines). (E) Three-dimensional renderings of four nematodes of which two were surrounded by a homogeneous deposit (cyan cloud). ( $F$ ) High-resolution MSI shows distribution of two metabolites, for orientation the bright-field image of section s3.2 shows the nematodes (magenta outlines) magnified in a cyst. The distributions of spermidine $\left(\mathrm{m} / \mathrm{z} 146.1645,\left[\mathrm{C}_{7} \mathrm{H}_{19} \mathrm{~N}_{3}+\mathrm{H}\right]^{+}\right)$and PAF $\left(\mathrm{m} / \mathrm{z} 482.3588,\left[\mathrm{C}_{24} \mathrm{H}_{52} \mathrm{NO}_{6} \mathrm{P}+\mathrm{H}\right]^{+}\right)$and an overlay of both metabolites is shown (PAF in cyan and spermidine in magenta). (G) Relative quantification of spermidine colocalized with nematode tissues (outlined in $F$ and $S I$ Appendix, Fig. S8) inside and outside the brown body cysts. cu, cuticle; nc, nematode cyst; vn, ventral nerve cord; $\mathrm{n}$, nematode; dep, deposit; bt, brown body tissue; gm, granular mass; i, electron dense inclusions. (Scale bars in C: $500 \mu \mathrm{m}$ and in $D-F: 50 \mu \mathrm{m}$.) 
densities of nematodes at an 11 times increased resolution $(0.325-\mu \mathrm{m}$ voxel size) in comparison to the resolution used for the $3 \mathrm{D}$ overview atlas (Fig. $3 A$ and $B$ ).

In the brown body tissue, SRmicro-CT revealed distinct histopathological states of the nematodes, only known from stereomicroscopic observations of live animals (41). We found nematodes that contained highly electron-dense inclusions and nematodes that were in the process of disintegrating into a granular mass, possibly induced by the earthworm's immune response (Fig. $3 \mathrm{D}$ and $E$ and SI Appendix, Fig. S6) (41). We also found histologically intact nematodes, some of which were surrounded by a homogeneous deposit (Fig. $3 D$ and $E$ ). This deposit was hypothesized to be a humoral response of the earthworm against the nematodes in the coelomic cavity (6).

The histology of these nematodes within the brown bodies was described in the 1970s. Today, our integrated micrometer-scale metabolite imaging provides insights into the potential metabolic function of the earthworm's humoral response and the nematodes' metabolic reaction. Our analysis of the 3D CHEMHIST atlas indicated a distinct tissue chemistry of the nematode cysts (Fig. 1B). Supporting a tissue-specific chemistry of the host-parasite interaction, the spatial clustering of the MSI data grouped a set of metabolite images (Fig. $1 F$ ) that only occurred in the brown bodies (see Fig. 1D).

To guide detailed metabolite imaging of single nematodes, we chose an adjacent tissue section with nematode cysts (Fig. $3 F$ and SI Appendix, Fig. S7). We imaged the encysted nematodes with a high-resolution MSI technique, termed atmospheric-pressure (AP) MALDI-orbitrap-MSI, that provided an $8-\mu \mathrm{m}$ pixel size, which is three times higher than the spatial resolution used in the $3 \mathrm{D}$ overview. For data analysis and exploration, we coregistered both high-resolution AP MALDI-orbitrap-MSI and SRmicro-CT datasets into the 3D atlas, extending the 3D model by different levels of resolution of the same structures (Fig. $3 A$ ).

The high-resolution metabolite images revealed that most nematodes were surrounded by a platelet activation factor (PAF), specifically lysophosphatidylcholine (lysoPC)O-16:0/0:0 (Fig. $3 F$ and SI Appendix, Fig. S7 and Table S1). PAFs are phospholipids with a single fatty acid chain and serve as inflammatory modulators, conserved in metazoans, across all domains of life (42). However, beyond the up- and down-regulation of PAF lipids as humoral immune response their site of production upon animalmicrobe and animal-parasite interactions remained unknown so far $(43,44)$. The PAF [(lysoPC)O-16:0/0:0] described here showed accumulations around the nematodes (Fig. $3 F$ ). This PAF is likely to promote the aggregation of hemocytes (45), which form most of the brown body tissue $(6,40)$ and release the reactive oxygen species (44). Although we hypothesize that the earthworm produces PAF lipids as an inflammatory response to the nematodes, from looking at only a snapshot of this metabolic interaction we cannot exclude that the PAFs originated from the nematodes. Nevertheless, we show that PAF lipids concentrate at the hostparasite interface, whereas they are absent in the nematode tissues but colocalize with aggregated hemocytes (SI Appendix, Fig. S7).

Focusing on the metabolite profiles of encysted nematodes, we detected twice as much of the polyamine spermidine compared to nematodes not within the brown bodies. Nematodes that were not encysted had spermidine signals as low as earthworm tissues (Fig. $3 G$ and SI Appendix, Figs. S7 and S8 and Table S1). Supplementing spermidine to other nematodes enhanced longevity, inducing autophagy and suppressing oxidative stress and necrosis (46). The nematodes encapsulated in brown bodies might increase their levels of spermidine to inhibit necrosis, as a protection against the reactive oxygen species of the earthworm. The production of spermidine as an antioxidative stress response could help some of the nematodes to survive in the brown bodies until the earthworm sheds its posterior segments (47), providing an escape mechanism from their host $(41,48)$.

\section{Conclusion}

Faced with the intimidating complexity of natural systems, scientists have studied model organisms under controlled conditions and thereby gained an understanding of their detailed molecular biology. This meticulous research on model organisms has created a strong foundation of databases and new technologies. Today, this groundwork allows us to address this complexity in naturally occurring symbioses and tease apart their metabolic interactions that promote phenotypic heterogeneity.

With CHEMHIST we present a cultivation-independent technique that does not require prior knowledge of the sample and can deliver unprecedented in situ visualizations of millimeter-sized animals and their (micro)organisms. To do so, we exclusively integrated ex vivo and in situ imaging techniques, limited to "snapshots" of a given state of a sample. Studying histological changes as a function of metabolic interactions between host and symbionts over time could be a major future avenue for CHEMHIST. This would require an anatomic and metabolic baseline that could be established through replication of our pipeline and allow comparisons between different life stages or colonization stages in symbioses.

Notably, CHEMHIST resembles an elaborate workflow that demands access to the different machines, which can be challenging for larger sample sizes in the range of hundreds of specimens. While high-resolution MSI and SRmicro-CT are still limited to specialized facilities, the laboratory-based MSI and micro-CT setups, which we used to generate the 3D atlas, have become standard equipment at imaging core facilities of most universities and research institutes. Currently, the acquisition, composition, and analysis of a CHEMHIST 3D atlas as presented in this study takes within 1 to 3 mo. The increased imaging speed and sensitivity of most current micro-CT and MSI technologies would allow the processing of tens of samples within a similar time frame, enabling the replication of our CHEMHIST approach.

Extending histotomography (26) into CHEMHIST opens nearly uncharted territory for label-free correlative imaging. Although our approach provided a wealth of biological and biochemical information, our approach of dividing the sample for the separate techniques led to a loss of 1 to $5 \%$ of the sample tissue that could have contained relevant details. Addressing this drawback, future technical developments of CHEMHIST could integrate phase-contrast SRmicro-CT (49), an emerging technique that allows quantitative $3 \mathrm{D}$ imaging of tissues without contrasting agents at nanometer scales $(50,51)$. Serial MSI after phase-contrast SRmicro-CT would provide data for a lossless anatomic and metabolic 3D model of the same organism (52) and the basis for machine learning-based correlations between modalities in 3D (53).

We envision that our advances in correlative chemical and structural in situ imaging will drive discovery-based research and fuel scientists' hypotheses on the metabolic interactions of their symbiotic systems.

\section{Materials and Methods}

Chemicals and Reagents. All chemicals were obtained from Sigma-Aldrich unless specified otherwise.

Tissues and Sample Collection. An adult earthworm (L. rubellus) (34) was collected from soil in a polder region of the central Netherlands (supplied by Lasebo BV) and rapidly frozen in isopentane cooled with liquid nitrogen. The posterior end (30 segments, $\sim 2 \mathrm{~cm}$ ) was embedded in $2 \%$ carboxymethylcellulose (CMC) gel and subsequently solidified at $-20{ }^{\circ} \mathrm{C}$. Using a precooled razor blade, the embedded earthworm was trimmed inside a cryochamber. Sectioning of the frozen sample block was performed as follows. The first tissue section ( 1 to $3 \mathrm{~mm}$ ) was cut with a razor blade from the frozen CMC block. The block was then trimmed and several $16-\mu \mathrm{m}$ sections were obtained with a cryotome $\left(-20{ }^{\circ} \mathrm{C}\right)$. This was repeated to obtain five blocks of tissue and four adjacent thin sections. Thin sections were transferred onto Bruker ITO glass slides via thaw mounting. Small crosses (1 to 2 
$\mathrm{mm}$ ) were drawn around the dried samples using a white paint marker (Edding 751) as a reference for the computational alignment (14). The slides were stored at $4{ }^{\circ} \mathrm{C}$ prior to MSI matrix application. The tissue blocks were used for micro-CT measurements and the adjacent thin sections for correlative bright-field microscopy, MSI, and FISH imaging.

Micro-CT. The frozen tissue blocks were defrosted in $8 \%$ paraformaldehyde. This chemical postcryo fixation with paraformaldehyde and osmium tetroxide allowed us to minimize the tissue damage induced by thawing and to preserve subcellular detail for high-resolution micro-CT. Contrasting was achieved using an aqueous $1 \%$ osmium tetroxide/acetone (1:1 vol/vol) solution for $2.5 \mathrm{~h}$ at $20{ }^{\circ} \mathrm{C}$. Dehydration and infiltration were conducted according to the 45,345 FLUKA Epoxy embedding medium data sheet. The resin blocks were pretrimmed with a fretsaw then fine-trimmed using a razor blade (54). Trimmed embedded earthworm tissue blocks were mounted individually on glass rods with a hot-melt gun. A Nanotom M computed tomography system (GE Measurement \& Control) was used for the micro-CT data acquisition of the tissue blocks with the following X-ray parameters: $110 \mathrm{kV}, 120 \mu \mathrm{A}, 0.75$-s exposure time, averaging $=4$, scanning time $=1.5 \mathrm{~h}$, number of projections acquired during scan $=1,500$. The tomographic reconstruction was performed using the phoenix datos $\mid x 2.2$ reconstruction software (GE Measurement \& Control) and resulted in a voxel size of $4.4 \mu \mathrm{m}$. The 16-bit volume was saved in *.vgl format. This format was imported into the 3D-visualization software VGStudio (2.2) (Volume Graphics) for cropping, histogram and bit dept (to 8 bit) adjustment, and data format (to *.raw format) conversion.

Synchrotron Radiation-Based Micro-Computed Phase-Contrast X-Ray Tomography P14 Beamline. After determining the position of the encysted nematodes in the laboratory-based micro-CT data, one small tissue block $(\sim 1 \times 1 \times 3 \mathrm{~mm})$ was cut out from the third tissue block with a razor blade in an area that contained the nematodes. This block was mounted onto a SPINE sample holder (55). Measurements were carried out on the European Molecular Biology Laboratory (EMBL) undulator beamline P14 at the PETRA-III storage ring (DESY at Hamburg Germany) using the propagation-based phase-contrast imaging setup described in refs. 56 and 57. X-ray energy of $18 \mathrm{keV}$ was used. The X-ray images were obtained using an X-ray microscope (Optique Peter) consisting of an LSO:Tb scintillator with a thin active layer of $8 \mu \mathrm{m}$, an Olympus UPlanFL 20-fold objective lens, a $45^{\circ}$-reflecting mirror, an Olympus $180-\mathrm{mm}$ tube lens, and a PCO.edge 4.2 scientific complementary metal-oxide-semiconductor sCMOS camera with a $2,048-\times 2,048$-pixel sensor with a pixel size of $6.5 \mu \mathrm{m}$. The effective pixel size of $0.325 \mu \mathrm{m}$ resulted in a $666-\times 666-\mu \mathrm{m}^{2}$ field of view. To ensure artifactless phase retrieval $(58)$ in the near-field edge-enhancing regime, each tomographic acquisition consisted of four measurements at sample-to-detector distances of 5.9, $6.4,7$, and $7.9 \mathrm{~cm}$. A total of 1,850 projections covering $185^{\circ}$ of continuous rotation and 40 flat-field images were acquired at each distance with a frame rate of 100 frames per second. A complete four-distance tomographic data acquisition took less than 2 min. Three measurements with overlapping areas were acquired along the vertically shifted sample to acquire the full length.

SRmicro-CT Data Processing. Data processing was carried out using in-house Python software, performing flat-field correction, phase retrieval, and tomographic reconstruction. First, each X-ray image of the sample was divided by the flat-field image with the highest similarity. For this operation, we used the similarity index (SSIM) implemented in the scikit-image Python module as a metric (59). Subsequently, a four-distance noniterative holographic reconstruction procedure $(60)$ was applied with a $\delta / \beta$ ratio of 0.17 to obtain a pro jected phase map of the sample at the given angle. The tomographic reconstruction was then performed using the tomopy Python module (61) with the gridrec algorithm and Shepp-Logan filter. The three reconstructed volumes were coregistered with the commercial software Amira 6.7.0 (Thermo Fisher Scientific), which did not require any nonlinear operations because we used the transform editor.

MALDI-TOF-MSI. For MALDI-MSI, a matrix consisting of $7 \mathrm{mg} \cdot \mathrm{mL}^{-1} \alpha$-cyano-4hydroxycinnamic acid in 70:30 acetonitrile/water with $0.2 \%$ trifluoroacetic acid was applied via an automated spray-coating system (SunCollect; SunChrom $\mathrm{GmbH}$ ) using the following parameters: $z$-distance of the capillary $25 \mathrm{~mm}$ and pressure of compressed air 2 bar; the flow for the first layer was $15 \mu \mathrm{L} \cdot \mathrm{min}^{-1}$ and for layers 2 to $820 \mu \mathrm{L} \cdot \mathrm{min}^{-1}$.

MALDI-MS imaging was performed using an Autoflex speed LRF MALDI-TOF (Bruker Daltonik) with MALDI Perpetual ion source and smartbeam-II $1 \mathrm{kHz}$ laser and reflector analysis in positive modes (18). A spot size of $25 \mu \mathrm{m}$ was used and 500 shots per sampling point were acquired using a "random walk" pattern with 100 shots per location within the sampling spot. The mass detection range was set to $\mathrm{m} / \mathrm{z} 100$ to 1,280 with 200 -ppm accuracy. For data processing and visualization of the MALDI-MS imaging data, flexImaging 4.0 (Bruker Daltonik) was used.

AP MALDI-Orbitrap-MSI. High-spatial-resolution MSI was performed using an atmospheric-pressure scanning microprobe MALDI source (AP SMALDI10; TransMIT GmbH) with a Q Exactive plus Fourier transform orbital trapping mass spectrometer (Thermo Scientific). A nitrogen laser with a wavelength of $337 \mathrm{~nm}$ and $60-\mathrm{Hz}$ repetition rate was used for desorption and ionization. The laser beam was focused to an $8-\mu \mathrm{m}$ ablation spot diameter and a step size of $8 \mu \mathrm{m}$ in $x$ and $y$ was used to scan the sample. Mass spectral acquisition was performed in positive mode and $\mathrm{m} / \mathrm{z} 100$ to $1,000 \mathrm{Da}$ with a mass resolving power of 140,000 (dataset in Fig. 3) at $\mathrm{m} / \mathrm{z} 200$ with a mass accuracy $<5 \mathrm{ppm}$. Additional datasets for the annotation of metabolites in METASPACE (62) and MS/MS experiments were recorded with a resolving power of 240,000 (SI Appendix, Table S1) at $\mathrm{m} / \mathrm{z} 200$ with a mass accuracy $<5$ ppm. The mass spectrometer was set to automatic gain control, fixed to 500-ms injection time. For data processing and visualization, ImageQuest 1.1 (Thermo Scientific) was used.

MALDI-MS ${ }^{2}$ The identification of spermidine and PAF was supported by MALDI-MS ${ }^{2}$ experiments (SI Appendix, Table S1 and Fig. S9). For PAF we obtained enough ions to use on tissue MALDI-MS ${ }^{2}$ in positive-ion mode with the mass analyzer set to a resolution of 240,000 at $\mathrm{m} / \mathrm{z} 200$ and a collision energy of $25 \mathrm{eV}$ in the higher-energy C-trap dissociation (HCD) cell. For spermidine, we could not obtain sufficient ions from the tissue to perform on tissue fragmentation. To support the identification of the exact mass, we matched the $\mathrm{MS}^{1} \mathrm{~m} / \mathrm{z}$ values of spermidine measured from the tissue with the $\mathrm{MS}^{1} \mathrm{~m} / \mathrm{z}$ values from a spermidine standard. The standard was spotted onto a glass slide and fragmented with MALDI-MS ${ }^{2}$, in positive-ion mode with the mass analyzer set to a resolution of 240,000 at $\mathrm{m} / \mathrm{z} 200$ and a collision energy of $30 \mathrm{eV}$ in the HCD cell.

MALDI-MSI Data Analysis and Visualization. The *.raw files were centroided and converted to *.mzML format with MSConvert GUI [ProteoWizard, version 3.0.9810 (63)] and then to *.imzML format using the imzML Converter 1.3 (64). SCiLS Lab software (SCiLS; Bruker Daltonik $\mathrm{GmbH}$ ) version $2019 \mathrm{~b}$ was used for spatial segmentation analysis and alignment of bright-field microscopy and MSI datasets as a template for selection of regions of interest.

DNA Extraction and Metagenomics Sequencing. Genomic DNA was extracted using the DNeasy Blood \& Tissue Kit (Qiagen). In brief, the nematode cysts from one consecutive tissue section of tissue section s3 (SI Appendix, Fig. S5) were scraped off the glass slide using a sterile scalpel and transferred into a tube containing $180 \mu \mathrm{L}$ buffer ATL and $20 \mu \mathrm{L}$ proteinase $\mathrm{K}$. The tissue was digested at $56{ }^{\circ} \mathrm{C}$ for $3 \mathrm{~d}$. Subsequent extraction steps were performed according to the manufacturer's instructions. In the end $100 \mu \mathrm{L}$ elution buffer were applied to the column and incubated at room temperature for $10 \mathrm{~min}$. After the first round of elution, a second elution $100 \mu \mathrm{L}$ of buffer was performed and the two elutions were pooled. The extracted DNA was stored at $4{ }^{\circ} \mathrm{C}$ until further processing.

Illumina-library preparation and sequencing were performed by the Max Planck Genome Centre. In brief, DNA quality was assessed with the Agilent 2,100 Bioanalyzer (Agilent) and genomic DNA was fragmented to an average fragment size of 400 base pairs (bp). An Illumina-compatible library was prepared using the TPase-based DNA library protocol. One nanogram of genomic DNA was cut and specific sequences were introduced by the Illumina Tagment DNA Enzyme (Illumina). Products were amplified by KAPA 2G Robust polymerase (Roche) with 15 cycles to enrich and to add libraryspecific barcoding information to the PCR products. After quality check by LabChip GX II (PerkinElmer) libraries were pooled and sequenced on an Illumina HiSeq 3000 sequencer with $2 \times 150$-bp paired-end mode. Three million 150-bp paired-end reads were sequenced on a HiSeq 3000 (Illumina).

Parasitic Nematode Phylogenetic Analyses Using the Small Subunit Ribosomal RNA Gene. We used phyloFlash v3.3 beta1 (https://github.com/HRGV/phyloFlash) (65) to assemble full-length small subunit (SSU) genes from the metagenomic reads. The nematode SSU matrix was constructed from the assembled Rhabditis related sequence, the available full-length SSU genes of all species level representatives of the Rhabditis group, and Teratorhabditis sequences as an outgroup. The sequences were aligned using MAFFT v7.394 (66) in G-Insi mode. The phylogenetic tree was reconstructed using FastTree v2.1.5 (67) with a GTR model, 20 rate categories, and Gamma20 likelihood optimization, generating approximate likelihood-ratio-test values for node support. The tree was drawn with Geneious R11 (https://www.geneious.com) and rooted with Teratorhabditis as an outgroup. 
Fluorescence In Situ Hybridization and Bright-Field Microscopy. After MALDIMS imaging, the matrix was removed by dipping the sample slide into $70 \%$ ethanol and $30 \%$ water (vol $/ \mathrm{vol}$ ) for $1 \mathrm{~min}$ each. In a second step, the tissue sections were postfixed for $1 \mathrm{~h}$ at $4{ }^{\circ} \mathrm{C}$ in $2 \%$ paraformaldehyde in phosphatebuffered saline (PBS). The sample was dried under ambient conditions and then prepared for catalyzed reporter deposition FISH following ref. 68. For in situ hybridization, general probes were used that target conserved regions of the $16 \mathrm{~S}$ ribosomal RNA in bacteria (EUB I to III; I: 5'-GCT GCC TCC CGT AGG AGT-3', II: 5'-GCA GCC ACC CGT AGG TGT-3', III: 5'-GCT GCC ACC CGT AGG TGT-3') $(69,70)$. To visualize tissue containing DNA, samples were stained for nuclei with DAPI for $10 \mathrm{~min}$ at room temperature, washed three times for $1 \mathrm{~min}$, and mounted in a VECTASHIELD/Citiflour mixture (2:11) with 1 part PBS (pH 9) under a coverslip (Menzel glass, $24 \times 60 \mathrm{~mm}$, \#1.5). Image acquisition for individual photomicrographs was carried out using a Zeiss Axioplan 2 microscope.

To image large sections for bright-field and fluorescence microscopy at high resolution an automated microscope (Zeiss Axio Imager Z2.m, 10x objective) with a tile-scan Macro for Axio Vision was used. Individual images were acquired with $15 \%$ overlap and stitched (Fiji Plugin stitching 1.1).

Composition of the 3D Atlas. To combine the different modalities into the 3D atlas, tools of Amira 6.7.0 were used for segmentation, surface rendering, and $3 \mathrm{D}$ coregistration (54). The 3D imaging platform AMIRA provided a graphical user interface (GUI) to visualize and coregister 3D and 2D imaging data (Movie S1). Using GUI-based software allows non-computer scientists to integrate their own correlative imaging data without programming and readily enables 3D data exploration and analysis.

The individual micro-CT volumes were imported as *.raw files and manually realigned, based on the estimates of the location and composition of shared morphological features, such as the overall number of the earthworm segments. Exact spacing of the intervals between tissue blocks relied on an estimate, as the precise thickness of tissue used for the sections could not be recorded due to loss of tissue during sectioning. Subsequently, the light

1. N. Fierer, Earthworms' place on Earth. Science 366, 425-426 (2019).

2. M. Delgado-Baquerizo et al., A global atlas of the dominant bacteria found in soil. Science 359, 320-325 (2018).

3. L. Tedersoo et al., FUNGAL BIOGEOGRAPHY. Response to comment on "Global diversity and geography of soil fungi". Science 349, 936 (2015).

4. J. van den Hoogen et al., Soil nematode abundance and functional group composition at a global scale. Nature 572, 194-198 (2019).

5. S. K. Davidson, D. A. Stahl, Transmission of nephridial bacteria of the earthworm Eisenia fetida. Appl. Environ. Microbiol. 72, 769-775 (2006).

6. G. O. Poinar, R. T. Hess, "Immune responses in the earthworm, Aporrectodea trapezoides (Annelida), against Rhabditis pellio (Nematoda)" in Comparative Pathobiology, L. A. Bulla, T. C. Cheng, Eds. (Springer, Boston, MA, 1977), vol. 3, pp. 69-84.

7. M. Liebeke et al., Unique metabolites protect earthworms against plant polyphenols. Nat. Commun. 6, 7869 (2015)

8. M. B. Lund, M. Holmstrup, B. A. Lomstein, C. Damgaard, A. Schramm, Beneficial effect of Verminephrobacter nephridial symbionts on the fitness of the earthworm Aporrectodea tuberculata. Appl. Environ. Microbiol. 76, 4738-4743 (2010).

9. G. O. Poinar, Associations between Nematodes (Nematoda) and Oligochaetes (Annelida). Proc. Helminthol. Soc. Wash. 45, 202-210 (1978).

10. H. R. P. Phillips et al., Global distribution of earthworm diversity. Science 366, 480-485 (2019).

11. C. Tropini, K. A. Earle, K. C. Huang, J. L. Sonnenburg, The gut microbiome: Connecting spatial organization to function. Cell Host Microbe. 21, 433-442 (2017).

12. V. V. Phelan, W. T. Liu, K. Pogliano, P. C. Dorrestein, Microbial metabolic exchange-The chemotype-to-phenotype link. Nat. Chem. Biol. 8, 26-35 (2011).

13. B. Geier et al., Spatial metabolomics of in situ host-microbe interactions at the micrometre scale. Nat. Microbiol. 5, 498-510 (2020).

14. M. Kaltenpoth, K. Strupat, A. Svatoš, Linking metabolite production to taxonomic identity in environmental samples by (MA)LDI-FISH. ISME J. 10, 527-531 (2016).

15. S. Moriano-Gutierrez et al., Critical symbiont signals drive both local and systemic changes in diel and developmental host gene expression. Proc. Natl. Acad. Sci. U.S.A. 116, 7990-7999 (2019)

16. H. Hulme et al., Microbiome-derived carnitine mimics as previously unknown mediators of gut-brain axis communication. Sci. Adv. 6, eaax6328 (2020).

17. A. D. Palmer, T. Alexandrov, Serial 3D imaging mass spectrometry at its tipping point Anal. Chem. 87, 4055-4062 (2015).

18. J. Oetjen et al., MRI-compatible pipeline for three-dimensional MALDI imaging mass spectrometry using PAXgene fixation. J. Proteomics 90, 52-60 (2013).

19. A. S. Attia et al., Monitoring the inflammatory response to infection through the integration of MALDI IMS and MRI. Cell Host Microbe. 11, 664-673 (2012).

20. J. E. Cassat et al., Integrated molecular imaging reveals tissue heterogeneity driving host-pathogen interactions. Sci. Transl. Med. 10, eaan6361 (2018).

21. T. K. Sinha et al., Integrating spatially resolved three-dimensional MALDI IMS with in vivo magnetic resonance imaging. Nat. Methods 5, 57-59 (2008) microscopy images were coregistered into the spaces between tissue blocks using morphologic structures in the orthographic cross-section of the micro-CT data as a reference. Based on the previous alignment of the 2D-imaging modalities in SCiLS Lab, the coregistration parameters could be applied to the FISH and MALDI-imaging data. All 3D graphical processes and applications with high computation demands were performed using a 3D imaging workstation (Windows 7 Professional, 64 bit, Intel Core i7-5960X central processing unit with 16 processors $\times 3.5 \mathrm{GHz}, 128 \mathrm{~GB}$ RAM, and NVIDIA Quadro P6000 with $24 \mathrm{~GB}$ )

Data Availability. All microscopy and (SR)micro-CT datasets can be directly downloaded from Figshare: laboratory-based micro-CT (https://doi.org/10.6084/m9 figshare.13011224), SRmicro-CT (https://doi.org/10.6084/m9.figshare.13011284), and bright-field and fluorescence microscopy (https://doi.org/10.6084/m9.figshare. 13011218). All MALDI-MSI and MALDI-MS/MS data were deposited within a project on the MetaboLights database under the accession number MTBLS2639. Additionally, the high-resolution MALDI-orbitrap-MSI data (MPIMM_017_QE_P_ LT) can be browsed on the online MSI annotation platform Metaspace (http:// www.metaspace2020.eu). The metagenomic sequencing data is available on the European Nucleotide Archive under accession number PRJEB45787.

ACKNOWLEDGMENTS. We thank Maggie E. Sogin (Max Planck Institute [MPI] Bremen) for constructive feedback and Janine Beckmann (MPI Bremen) for help with MALDI-MSI and MS/MS experiments and Miriam Sadowski (MPI Bremen) for help with DNA extractions. We thank Gleb Bourenkov (European Molecular Biology Laboratory Hamburg) for the valuable support in X-ray experiments at the P14 beamline. We thank Russell Naisbit, Wolfgang Geier, and Grace D'Angelo for additional edits of the manuscript draft. We thank Theodore Alexandrov and Kathrin Maedler (University of Bremen) for providing access to MALDI TOF instrumentation. We thank Nicole Dubilie (MPI Bremen) for giving access to resources and for constructive feedback. This work was funded by the Gordon and Betty Moore Foundation Marine Microbiology Initiative Investigator Award (Grant GBMF3811) and the Max Planck Society.

22. A. E. Douglas, Simple animal models for microbiome research. Nat. Rev. Microbiol. 17, 764-775 (2019).

23. S. J. Van Malderen et al., Three-dimensional reconstruction of the tissue-specific multielemental distribution within Ceriodaphnia dubia via multimodal registration using laser ablation ICP-mass spectrometry and x-ray spectroscopic techniques. Anal. Chem. 89, 4161-4168 (2017).

24. M. Töpperwien, F. van der Meer, C. Stadelmann, T. Salditt, Three-dimensional virtual histology of human cerebellum by x-ray phase-contrast tomography. Proc. Natl. Acad. Sci. U.S.A. 115, 6940-6945 (2018)

25. M. Müller et al., Myoanatomy of the velvet worm leg revealed by laboratory-based nanofocus X-ray source tomography. Proc. Natl. Acad. Sci. U.S.A. 114, 12378-12383 (2017).

26. Y. F. Ding et al., Computational 3D histological phenotyping of whole zebrafish by X-ray histotomograhpy. eLife 8, e44898 (2019).

27. M. Busse et al., Three-dimensional virtual histology enabled through cytoplasmspecific X-ray stain for microscopic and nanoscopic computed tomography. Proc. Natl. Acad. Sci. U.S.A. 115, 2293-2298 (2018).

28. M. Kompauer, S. Heiles, B. Spengler, Atmospheric pressure MALDI mass spectrometry imaging of tissues and cells at 1.4- $\mu \mathrm{m}$ lateral resolution. Nat. Methods 14, 90-96 (2017).

29. M. Niehaus, J. Soltwisch, M. E. Belov, K. Dreisewerd, Transmission-mode MALDI-2 mass spectrometry imaging of cells and tissues at subcellular resolution. Nat. Methods 16, 925-931 (2019).

30. R. Fernández, S. Kvist, J. Lenihan, G. Giribet, A. Ziegler, Sine systemate chaos? A versatile tool for earthworm taxonomy: Non-destructive imaging of freshly fixed and museum specimens using micro-computed tomography. PLoS One 9, e96617 (2014).

31. X. Xu et al., A brief review on mass/optical spectrometry for imaging analysis of biological samples. Appl. Spectrosc. Rev. 54, 57-85 (2019)

32. J. M. Spraggins et al., Next-generation technologies for spatial proteomics: Integrating ultra-high speed MALDI-TOF and high mass resolution MALDI FTICR imaging mass spectrometry for protein analysis. Proteomics 16, 1678-1689 (2016).

33. N. Verbeeck et al., Connecting imaging mass spectrometry and magnetic resonance imaging-based anatomical atlases for automated anatomical interpretation and differential analysis. Biochim. Biophys. Acta. Proteins Proteomics 1865, 967-977 (2017).

34. W. Hoffmeister, Beitrag zur Kenntnis deutscher Landanneliden. Arch. Naturgesch. 9 183-198 (1843)

35. H. Thiele et al., 2D and 3D MALDI-imaging: Conceptual strategies for visualization and data mining. Biochim. Biophys. Acta 1844 (1 Pt A), 117-137 (2014).

36. J. Roche, J. Roche; van THOAl, Phosphagens of marine animals. Ann. N. Y. Acad. Sci. 90, 923-928 (1960)

37. A. E. Needham, Distribution of protoporphyrin, ferrihaem and indoles in the body wall of L. terrestris. Comp. Biochem. Physiol. 26, 429-442 (1968).

38. C. G. Goodchild, G. H. Irwin, Occurrence of Nematodes Rhabditis anomala and $R$ pellio in Oligochaetes Lumbricus rubellus and L. terrestris. Trans. Am. Microsc. Soc. $\mathbf{9 0}$ 231-237 (1971) 
39. P. Valembois, J. Seymour, M. Lassègues, Evidence of lipofuscin and melanin in the brown body of the earthworm Eisenia fetida andrei. Cell Tissue Res. 277, 183-188 (1994)

40. P. Valembois, M. Lassègues, P. Roch, Formation of brown bodies in the coelomic cavity of the earthworm Eisenia fetida andrei and attendant changes in shape and adhesive capacity of constitutive cells. Dev. Comp. Immunol. 16, 95-101 (1992).

41. G. O. Poinar, G. M. Thomas, Rhabditis pellio Schneider (nematoda) from the earth worm, Aporrectodea trapezoides Duges (Annelida). J. Nematol. 7, 374-379 (1975)

42. T. Sugiura, T. Fukuda, T. Miyamoto, K. Waku, Distribution of alkyl and alkenyl etherlinked phospholipids and platelet-activating factor-like lipid in various species of in vertebrates. Biochim. Biophys. Acta 1126, 298-308 (1992)

43. T. Sugiura et al., Platelet-activating factor and its structural analogues in the earth worm Eisenia foetida. Biochim. Biophys. Acta 1258, 19-26 (1995).

44. R. A. Quinn et al., Correction to 'Metabolomics of reef benthic interactions reveals a bioactive lipid involved in coral defence'. Proc. Biol. Sci. 283, 20160469 (2016).

45. E. S. Garcia, D. P. Castro, M. B. Figueiredo, F. A. Genta, P. Azambuja, Trypanosoma rangeli: A new perspective for studying the modulation of immune reactions of Rhodnius prolixus. Parasit. Vectors 2, 33 (2009).

46. T. Eisenberg et al., Induction of autophagy by spermidine promotes longevity. Nat Cell Biol. 11, 1305-1314 (2009)

47. D. Keilin, Parasitic autotomy of the host as a mode of liberation of coelomic parasites from the body of the earthworm. Parasitology 17, 170-172 (1925).

48. J. A. Somers, H. H. Shorey, L. K. Gaston, Reproductive biology and behavior of Rhabditis pellio, (Schneider) (Rhabditida: Rhabditidae). J. Nematol. 9, 143-148 (1977).

49. A. Snigirev, I. Snigireva, V. Kohn, S. Kuznetsov, I. Schelokov, On the possibilities of $x$ ray phase contrast microimaging by coherent high-energy synchrotron radiation. $R e v$. Sci. Instrum. 66, 5486-5492 (1995).

50. O. Betz et al., Imaging applications of synchrotron X-ray phase-contrast micro tomography in biological morphology and biomaterials science. I. General aspects of the technique and its advantages in the analysis of millimetre-sized arthropod structure. J. Microsc. 227, 51-71 (2007).

51. J. Herzen et al., 3D grating-based X-ray phase-contrast computed tomography for high-resolution quantitative assessment of cartilage: An experimental feasibility study with 3T MRI, 7T MRI and biomechanical correlation. PLoS One 14, e0212106 (2019).

52. B. Geier et al., "Correlative 3D anatomy and spatial chemistry in animalmicrobe symbioses: Developing sample preparation for phase-contrast synchrotron radiation based micro-computed tomography and mass spectrometry imaging" in Develop ments in X-Ray Tomography XII, B. Müller, Ed. (SPIE, 2019), 11113, p. 1111306.

53. R. Van de Plas, J. Yang, J. Spraggins, R. M. Caprioli, Image fusion of mass spectrometry and microscopy: A multimodality paradigm for molecular tissue mapping. Nat Methods 12, 366-372 (2015).
54. S. Handschuh, N. Baeumler, T. Schwaha, B. Ruthensteiner, A correlative approach for combining microCT, light and transmission electron microscopy in a single 3D scenario. Front. Zool. 10, 44 (2013).

55. F. Cipriani et al., Automation of sample mounting for macromolecular crystallography. Acta Crystallogr. D Biol. Crystallogr. 62, 1251-1259 (2006).

56. M. Polikarpov et al., Visualization of protein crystals by high-energy phase-contrast X-ray imaging. Acta Crystallogr. D Struct. Biol. 75, 947-958 (2019).

57. M. Polikarpov, G. Bourenkov, A. Snigirev, T. Schneider, "High-throughput x-ray imaging, microscopy, and tomography for biological applications on EMBL beamline P14 at PETRA III (conference presentation)" in Developments in XRay Tomography XII, B. Müller, Ed. (SPIE, 2019), 11113 , p. 1111307.

58. S. Zabler, P. Cloetens, J. P. Guigay, J. Baruchel, M. Schlenker, Optimization of phase contrast imaging using hard $\mathrm{x}$ rays. Rev. Sci. Instrum. 76, 073705 (2005).

59. Z. Wang, A. C. Bovik, H. R. Sheikh, E. P. Simoncelli, Image quality assessment: From error visibility to structural similarity. IEEE Trans. Image Process. 13, 600-612 (2004).

60. P. Cloetens et al., Holotomography: Quantitative phase tomography with micrometer resolution using hard synchrotron radiation x rays. Appl. Phys. Lett. 75, 2912-2914 (1999).

61. D. Gürsoy, F. De Carlo, X. Xiao, C. Jacobsen, TomoPy: A framework for the analysis of synchrotron tomographic data. J. Synchrotron Radiat. 21, 1188-1193 (2014).

62. T. Alexandrov et al., METASPACE: A community-populated knowledge base of spatial metabolomes in health and disease. bioRxiv [Preprint] (2019). https://doi.org/10.1101/ 539478 (Accessed 17 June 2021)

63. M. C. Chambers et al., A cross-platform toolkit for mass spectrometry and proteomics. Nat. Biotechnol. 30, 918-920 (2012).

64. A. M. Race, I. B. Styles, J. Bunch, Inclusive sharing of mass spectrometry imaging data requires a converter for all. J. Proteomics 75, 5111-5112 (2012).

65. H. R. Gruber-Vodicka, B. K. B. Seah, E. Pruesse, phyloFlash: Rapid small-subunit rRNA profiling and targeted assembly from metagenomes. mSystems 5, 10.1128/mSystems.00920-20 (2020).

66. K. Katoh, D. M. Standley, MAFFT multiple sequence alignment software version 7: Improvements in performance and usability. Mol. Biol. Evol. 30, 772-780 (2013).

67. M. N. Price, P. S. Dehal, A. P. Arkin, FastTree 2-Approximately maximum-likelihood trees for large alignments. PLoS One 5, e9490 (2010).

68. A. Pernthaler, J. Pernthaler, R. Amann, Fluorescence in situ hybridization and catalyzed reporter deposition for the identification of marine bacteria. Appl. Environ. Microbiol. 68, 3094-3101 (2002).

69. R. I. Amann et al., Combination of $16 \mathrm{~S}$ rRNA-targeted oligonucleotide probes with flow cytometry for analyzing mixed microbial populations. Appl. Environ. Microbiol. 56, 1919-1925 (1990).

70. H. Daims, A. Brühl, R. Amann, K. H. Schleifer, M. Wagner, The domain-specific probe EUB338 is insufficient for the detection of all Bacteria: Development and evaluation of a more comprehensive probe set. Syst. Appl. Microbiol. 22, 434-444 (1999).
Geier et al.

Connecting structure and function from organisms to molecules in small-animal symbioses through chemo-histo-tomography
PNAS | 9 of 9 